\title{
Article \\ Coded Control of a Sectional Electroelastic Engine for Nanomechatronics Systems
}

\author{
Sergey M. Afonin
}

check for updates

Citation: Afonin, S.M. Coded Control of a Sectional Electroelastic Engine for Nanomechatronics Systems. Appl. Syst. Innov. 2021, 4, 47. https://doi.org/10.3390/asi4030047

Academic Editors:

Emmanuel Karapidakis and Teen Hang Meen

Received: 20 March 2021

Accepted: 23 July 2021

Published: 28 July 2021

Publisher's Note: MDPI stays neutral with regard to jurisdictional claims in published maps and institutional affiliations.

Copyright: (C) 2021 by the author. Licensee MDPI, Basel, Switzerland. This article is an open access article distributed under the terms and conditions of the Creative Commons Attribution (CC BY) license (https:// creativecommons.org/licenses/by/ $4.0 /)$.
National Research University of Electronic Technology (MIET), 124498 Moscow, Russia; learner01@mail.ru

\begin{abstract}
This work determines the coded control of a sectional electroelastic engine at the elasticinertial load for nanomechatronics systems. The expressions of the mechanical and adjustment characteristics of a sectional electroelastic engine are obtained using the equations of the electroelasticity and the mechanical load. A sectional electroelastic engine is applied for coded control of nanodisplacement as a digital-to-analog converter. The transfer function and the transient characteristics of a sectional electroelastic engine at elastic-inertial load are received for nanomechatronics systems.
\end{abstract}

Keywords: sectional electroelastic engine; coded control; sectional piezoelectric engine; mechanical and adjustment characteristics; elastic-inertial load; transfer function; transient characteristic; nanomechatronics system

\section{Introduction}

The application of a multilayer electroelastic engine, a compound electroelastic engine, and a sectional electroelastic engine on the piezoelectric effect or the electrostriction effect are promising for nanomanipulators in nanotechnology [1-3]. To increase the range of the displacement, a multilayer electroelastic engine and a sectional electroelastic engine are used $[4,5]$. A multilayer electroelastic engine has lower control voltage, making it safer to use. Its piezolayers can be combined into sections [6]. A multilayer sectional electroelastic engine is used in nanotechnology and nanobiomedicine, adaptive optics, interferometers and laser research [7]. A sectional electroelastic engine solves tasks of precise nanopositioning adjustment for nanotechnology, adaptive optics, antennas and nanomechatronics systems [7-9].

This work uses the separation of an electroelastic engine into several sections to achieve more precise control and two-level voltage switching. The piezolayers are connected in parallel in each section. The number of piezolayers in a section is equal to 2 in the power of $0,1,2,3,4$, and so on. A sectional electroelastic engine operates as a digital-to-analog converter. The tasks of providing the accuracy and speed of the control system using a nanopositioning sectional electroelastic engine with the coded control are topical. Studies of the mechanical and adjustment characteristics of a nanopositioning sectional electroelastic engine with the coded control are relevant for nanotechnology. In the scanning probe microscope and the gene manipulator, a sectional electroelastic engine is applied for the scanner and the nanomanipulator $[7,10]$.

For nanomechatronics systems, the mechanical and adjustment characteristics of a sectional electroelastic engine are obtained. The transfer function and the transient response of a sectional electroelastic engine at elastic-inertial load are received.

\section{Mechanical and Adjustment Characteristics of a Sectional Electroelastic Engine}

Let us consider the mechanical and adjustment characteristics in the static of a sectional electroelastic engine for nanomechatronics systems at the transverse, longitudinal and shift piezoeffects. The piezolayers in a sectional electroelastic engine are connected in parallel in each section of a sectional electroelastic engine. 
The control voltage is supplied to each section via the multiplexer. Several sections are connected via the multiplexer two-level control voltage in the forms 0 or $U$. In terms of statics, each section is deformed, and the movement of the section takes a minimum value at 0 voltage or a maximum value at $U$ voltage.

A sectional electroelastic engine is used as a digital-to-analog converter-engine for nanodisplacement. The number of the piezolayers in the section is equal to the power of 2. To calculate the deformation of a sectional electroelastic engine, using the equations of the electroelasticity and the mechanical load is necessary. A sectional electroelastic engine consists of $N$ sections with all $n$ layers.

For a sectional electroelastic engine, the equation of the electroelasticity [10-29] has the form of the inverse piezoelectric effect

$$
S_{i}=d_{m i} E_{m}+s_{i j}^{E} T_{j}
$$

where $S_{i}, d_{m i}, E_{m}, s_{i j}^{E}$, and $T_{j}$ are the relative deformation, the coefficient of electroelasticity, the electric field strength, the elastic compliance with $E=$ const and the mechanical tension, respectively, with the indices $i, j=1,2, \ldots, 6, m=1,2,3$.

The mechanical characteristic of a sectional electroelastic engine has the form

$$
\Delta l(F)=\left.\Delta l\left(U, a_{k}, F\right)\right|_{U=\text { const }, a_{k}=\text { const }}
$$

where $\Delta l, F, U$, and $a_{k}$ are the deformation, the force, the voltage and the binary code for the $k$ section, respectively.

The adjustment characteristic of a sectional electroelastic engine at elastic load has the form

$$
\Delta l(U)=\left.\Delta l\left(U, a_{k}, F\right)\right|_{F=C_{e} \Delta l, C_{e}=\text { const }, a_{k}=\text { const }}
$$

where $C_{e}$ is the rigidity of the load.

For a sectional piezoelectric engine at the transverse piezoeffect on Figure 1, the equation of the electroelasticity [6-15] has the form

$$
S_{1}=d_{31} E_{3}+s_{11}^{E} T_{1}
$$

where $S_{1}, d_{31}, E_{3}, s_{11}^{E}$, and $T_{1}$ are the relative deformation along axis 1 , the transverse piezomodule, the electric field strength along axis 3 on Figure 1, the elastic compliance with $E=$ const and the mechanical tension along axis 1 , respectively.

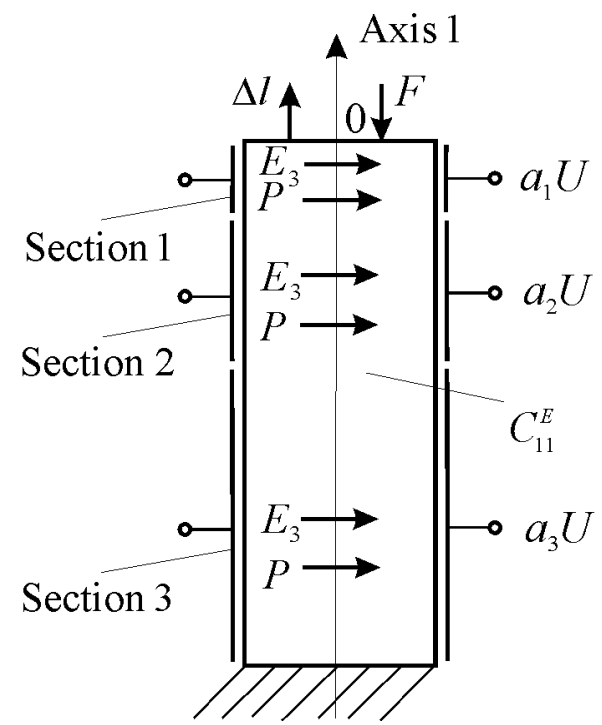

Figure 1. Sectional transverse piezoelectric engine for nanomechatronics systems. 
The displacement of the first section, a sectional piezoelectric engine at the transverse piezoeffect, has the form

$$
\Delta l_{1}=\frac{d_{31} l_{1} U}{\delta}
$$

where $l_{1}$ is the length of the first section engine and $\delta$ is the thickness.

The displacement $\Delta l_{k}$ of the $k$ section, a sectional piezoelectric engine at the transverse piezoeffect for $F=0$ from (5), has the form

$$
\Delta l_{k}=2^{k-1} \frac{d_{31} l_{1} U}{\delta}=2^{k-1} \Delta l_{1}=\frac{d_{31} l_{k} U}{\delta}
$$

The displacement of a sectional piezoelectric engine at the transverse piezoeffect on Figure 1 for $F=0$ from (6) is obtained in the form

$$
\Delta l=\frac{d_{31} l_{1} U}{\delta}\left(\sum_{k=1}^{N} a_{k} 2^{k-1}\right)=\Delta l_{1}\left(\sum_{k=1}^{N} a_{k} 2^{k-1}\right)
$$

where $a_{k}$ is the binary code for the $k$ section engine and $l=\sum_{k=1}^{N} l_{k}$ is the length of a sectional piezoelectric engine.

The mechanical characteristic of a sectional piezoelectric engine has the form

$$
\Delta l=\Delta l_{\max }\left(1-\frac{F}{F_{\max }}\right)
$$

where $\Delta l_{\max }$ and $F_{\max }$ are the maximum displacement of a sectional piezoelectric engine at $F=0$ and the maximum force at $\Delta l=0$, respectively.

The mechanical characteristic of a sectional piezoelectric engine at the transverse piezoeffect has the form

$$
\Delta l=\frac{d_{31} l_{1} U}{\delta}\left(\sum_{k=1}^{N} a_{k} 2^{k-1}\right)-\frac{s_{11}^{E} l F}{S_{0}}
$$

consequently

$$
\Delta l=\frac{d_{31} l_{1} U}{\delta}\left(\sum_{k=1}^{N} a_{k} 2^{k-1}\right)-\frac{F}{C_{11}^{E}}
$$

where $C_{11}^{E}=\frac{S_{0}}{s_{11}^{E} l}$ is the rigidity of a sectional piezoelectric engine at the transverse piezoeffect and $l$ is the length of a sectional transverse piezoelectric engine.

The maximum displacement and the maximum force at the transverse piezoeffect from the mechanical characteristic (8) have the form

$$
\begin{gathered}
\Delta l_{\max }=\frac{d_{31} l_{1} U}{\delta}\left(\sum_{k=1}^{N} a_{k} 2^{k-1}\right)=\Delta l_{1}\left(\sum_{k=1}^{N} a_{k} 2^{k-1}\right) \\
F_{\max }=\frac{d_{31} l_{1} U}{\delta}\left(\sum_{k=1}^{N} a_{k} 2^{k-1}\right) C_{11}^{E}
\end{gathered}
$$

For a sectional transverse piezoelectric engine from ceramic PZT at $d_{31}=0.2 \mathrm{~nm} / \mathrm{V}$, $l_{1} / \delta=1$, and $U=100 \mathrm{~V}$ for $a_{1}=1, a_{2}=0, a_{3}=0 ; a_{1}=1, a_{2}=1, a_{3}=0 ;$ and $a_{1}=1$, $a_{2}=1, a_{3}=1$, the maximum displacements of a sectional piezoelectric engine are obtained: $\Delta l_{\max }=20 \mathrm{~nm} ; \Delta l_{\max }=60 \mathrm{~nm} ;$ and $\Delta l_{\max }=140 \mathrm{~nm}$. 
The adjustment characteristic of a sectional piezoelectric engine at the transverse piezoeffect and elastic load has the form

$$
\Delta l=\frac{d_{31} l_{1} U}{\delta}\left(\sum_{k=1}^{N} a_{k} 2^{k-1}\right)-\frac{C_{e} \Delta l}{C_{11}^{E}}
$$

consequently

$$
\Delta l=\frac{\left(d_{31} l_{1} / \delta\right) U}{\left(1+C_{e} / C_{11}^{E}\right)}\left(\sum_{k=1}^{N} a_{k} 2^{k-1}\right)=\Delta l_{1}\left(\sum_{k=1}^{N} a_{k} 2^{k-1}\right)
$$

The displacement of the first section engine at the elastic load has the form

$$
\Delta l_{1}=\frac{\left(d_{31} l_{1} / \delta\right) U}{1+C_{e} / C_{11}^{E}}
$$

where $C_{e}$ and $C_{11}^{E}$ are the rigidity of the load and a sectional piezoelectric engine, respectively.

For a sectional piezoelectric engine at the longitudinal piezoeffect, the equation of the electroelasticity $[6-15,28]$ has the form

$$
S_{3}=d_{33} E_{3}+s_{33}^{E} T_{3}
$$

where $S_{3}, d_{33}, E_{3}, s_{33}^{E}$ and $T_{3}$ are the relative deformation along axis 3 , the longitudinal piezomodule, the electric field strength along axis 3 on Figure 2, the elastic compliance with $E=$ const and the mechanical tension along axis 3 , respectively.

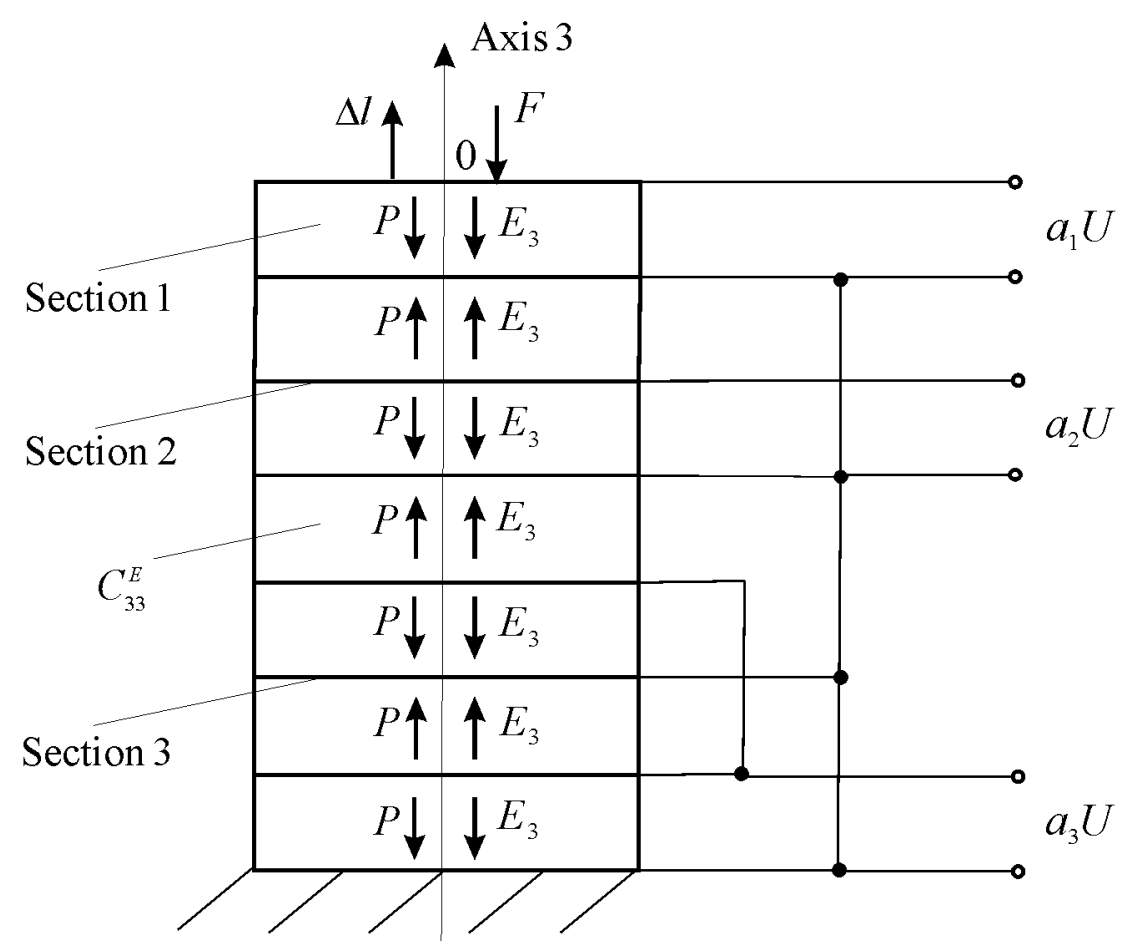

Figure 2. Sectional longitudinal piezoelectric engine for nanomechatronics systems.

For the mechanical characteristic (8) of a sectional piezoelectric engine at the longitudinal piezoeffect, the maximum displacement and the maximum force are obtained in the form

$$
\Delta l_{\max }=\frac{d_{33} l_{1} U}{\delta}\left(\sum_{k=1}^{N} a_{k} 2^{k-1}\right)=\Delta l_{1}\left(\sum_{k=1}^{N} a_{k} 2^{k-1}\right)
$$




$$
\begin{gathered}
F_{\max }=\frac{d_{33} l_{1} U}{\delta}\left(\sum_{k=1}^{N} a_{k} 2^{k-1}\right) C_{33}^{E} \\
\Delta l_{1}=\frac{d_{33} l_{1} U}{\delta}
\end{gathered}
$$

where $C_{33}^{E}=S_{0} /\left(s_{33}^{E} l\right)$ is the rigidity of a sectional piezoelectric engine at the longitudinal piezoeffect.

For a sectional longitudinal piezoelectric engine from ceramic PZT at $d_{33}=0.4 \mathrm{~nm} / \mathrm{V}$, $l_{1} / \delta=1, C_{33}^{E}=8 \times 10^{8} \mathrm{~N} / \mathrm{m}$, and $U=100 \mathrm{~V}$ for $a_{1}=1, a_{2}=0, a_{3}=0 ; a_{1}=1, a_{2}=1, a_{3}=0$; and $a_{1}=1, a_{2}=1, a_{3}=1$, the maximum displacements and the maximum forces of a sectional piezoelectric engine on Figure 3 are obtained (a) $\Delta l_{\max }=40 \mathrm{~nm}, F_{\max }=32 \mathrm{~N}$; (b) $\Delta l_{\max }=120 \mathrm{~nm}, F_{\max }=96 \mathrm{~N}$; and (c) $\Delta l_{\max }=280 \mathrm{~nm}, F_{\max }=224 \mathrm{~N}$.

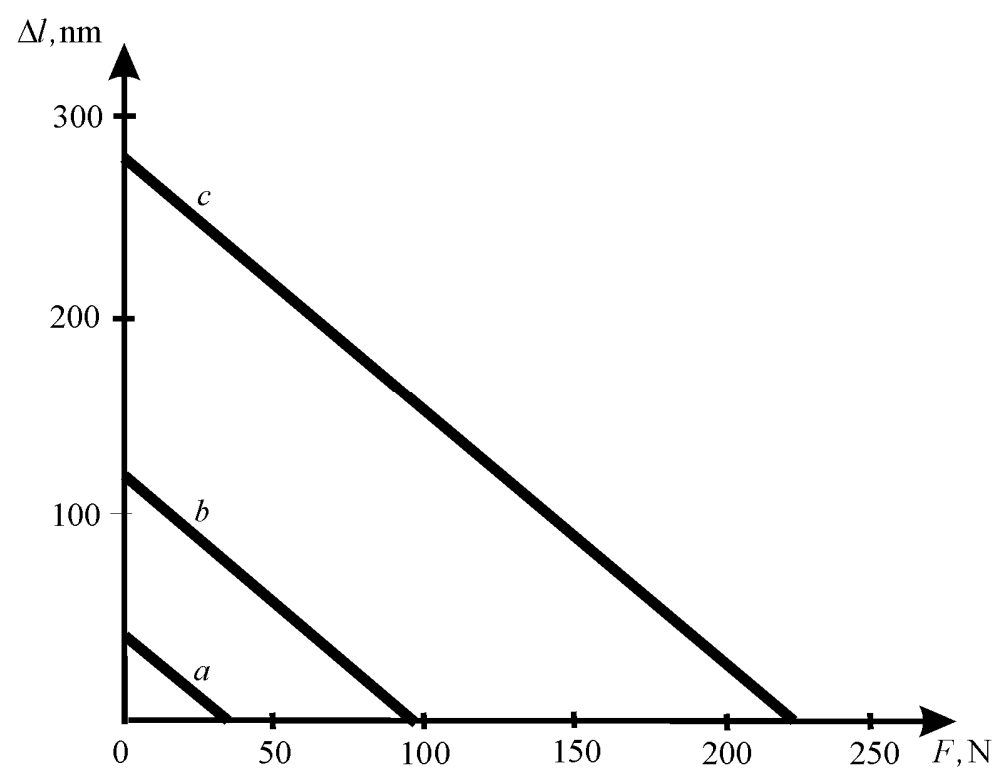

Figure 3. Mechanical characteristic of sectional longitudinal piezoelectric engine.

The adjustment characteristic of a sectional piezoelectric engine at the longitudinal piezoeffect and elastic load has the form

$$
\Delta l=\frac{d_{33} l_{1} U}{\delta}\left(\sum_{k=1}^{N} a_{k} 2^{k-1}\right)-\frac{C_{e} \Delta l}{C_{33}^{E}}
$$

consequently

$$
\begin{gathered}
\Delta l=\frac{\left(d_{33} l_{1} / \delta\right) U}{\left(1+C_{e} / C_{33}^{E}\right)}\left(\sum_{k=1}^{N} a_{k} 2^{k-1}\right)=\Delta l_{1}\left(\sum_{k=1}^{N} a_{k} 2^{k-1}\right) \\
\Delta l_{1}=\frac{\left(d_{33} l_{1} / \delta\right) U}{1+C_{e} / C_{33}^{E}}
\end{gathered}
$$

where $C_{33}^{E}=\frac{S_{0}}{s_{33}^{E}}$ is the rigidity of a sectional piezoelectric engine at the longitudinal piezoeffect.

For a sectional piezoelectric engine at the shift piezoeffect, the equation of the electroelasticity [6-15] has the form

$$
S_{5}=d_{15} E_{1}+s_{55}^{E} T_{5}
$$

where $S_{5}, d_{15}, E_{1}, s_{55}^{E}$, and $T_{5}$ are the relative deformation along axis 5 , the shift piezomodule, the electric field strength along axis 1 on Figure 4, the elastic compliance with $E=$ const and the mechanical shift tension along axis 5 , respectively. 


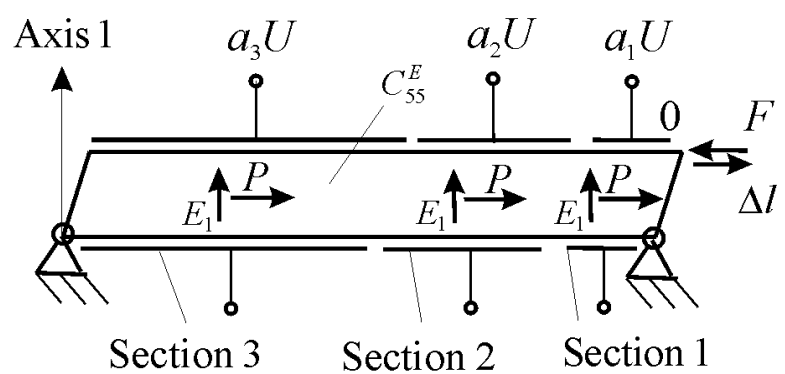

Figure 4. Sectional shift piezoelectric engine for nanomechatronics systems.

The maximum displacement and the maximum force at the shift piezoeffect from the mechanical characteristic (8) have the form

$$
\begin{gathered}
\Delta l_{\max }=\frac{d_{15} l_{1} U}{\delta}\left(\sum_{k=1}^{N} a_{k} 2^{k-1}\right)=\Delta l_{1}\left(\sum_{k=1}^{N} a_{k} 2^{k-1}\right) \\
F_{\max }=\frac{d_{15} l_{1} U}{\delta}\left(\sum_{k=1}^{N} a_{k} 2^{k-1}\right) C_{55}^{E} \\
\Delta l_{1}=\frac{d_{15} l_{1} U}{\delta}
\end{gathered}
$$

where $C_{55}^{E}=\frac{S_{0}}{S_{55}^{E} l}$ is the rigidity of a sectional piezoelectric engine at the shift piezoeffect.

For a sectional shift piezoelectric engine from ceramic PZT at $d_{15}=0.5 \mathrm{~nm} / \mathrm{V}, l_{1} / \delta=1$, and $U=100 \mathrm{~V}$ for $a_{1}=1, a_{2}=0, a_{3}=0 ; a_{1}=1, a_{2}=1, a_{3}=0$; and $a_{1}=1, a_{2}=1, a_{3}=1$ the maximum displacements of a sectional piezoelectric engine are received: $\Delta l_{\max }=50 \mathrm{~nm}$; $\Delta l_{\max }=150 \mathrm{~nm}$; and $\Delta l_{\max }=350 \mathrm{~nm}$.

The adjustment characteristic of a sectional piezoelectric engine at the shift piezoeffect and elastic load has the form

$$
\Delta l=\frac{d_{15} l_{1} U}{\delta}\left(\sum_{k=1}^{N} a_{k} 2^{k-1}\right)-\frac{C_{e} \Delta l}{C_{55}^{E}}
$$

consequently

$$
\begin{gathered}
\Delta l=\frac{\left(d_{15} l_{1} / \delta\right) U}{\left(1+C_{e} / C_{55}^{E}\right)}\left(\sum_{k=1}^{N} a_{k} 2^{k-1}\right)=\Delta l_{1}\left(\sum_{k=1}^{N} a_{k} 2^{k-1}\right) \\
\Delta l_{1}=\frac{\left(d_{15} l_{1} / \delta\right) U}{1+C_{e} / C_{55}^{E}}
\end{gathered}
$$

For the mechanical characteristic (8) of a sectional electroelastic engine, the maximum displacement and the maximum force are received in the form

$$
\begin{gathered}
\Delta l_{\max }=\frac{d_{m i} l_{1} U}{\delta}\left(\sum_{k=1}^{N} a_{k} 2^{k-1}\right)=\Delta l_{1}\left(\sum_{k=1}^{N} a_{k} 2^{k-1}\right) \\
F_{\max }=\frac{d_{m i} l_{1} U}{\delta}\left(\sum_{k=1}^{N} a_{k} 2^{k-1}\right) C_{i j}^{E} \\
\Delta l_{1}=\frac{d_{m i} l_{1} U}{\delta} \\
l=\sum_{k=1}^{N} l_{k}=\left(2^{N}-1\right) l_{1}
\end{gathered}
$$


where $C_{i j}^{E}=\frac{S_{0}}{s_{i j}^{E l}}$ is the rigidity of a sectional electroelastic engine and $l$ is the length of a sectional electroelastic engine.

The adjustment characteristic of a sectional electroelastic engine with the $N$ sections at elastic load is obtained in the form

$$
\Delta l=\frac{d_{m i} l_{1} U}{\delta}\left(\sum_{k=1}^{N} a_{k} 2^{k-1}\right)-\frac{C_{e} \Delta l}{C_{i j}^{E}}
$$

consequently

$$
\begin{gathered}
\Delta l=k_{U} U=\frac{\left(d_{m i} l_{1} / \delta\right) U}{\left(1+C_{e} / C_{i j}^{E}\right)}\left(\sum_{k=1}^{N} a_{k} 2^{k-1}\right)=\Delta l_{1} c \\
k_{U}=\frac{\left(d_{m i} l_{1} / \delta\right)}{\left(1+C_{e} / C_{i j}^{E}\right)}\left(\sum_{k=1}^{N} a_{k} 2^{k-1}\right), \Delta l_{1}=\frac{\left(d_{m i} l_{1} / \delta\right) U}{1+C_{e} / C_{i j}^{E}}, c=\sum_{k=1}^{N} a_{k} 2^{k-1}
\end{gathered}
$$

where $k_{U}$ is the transfer coefficient on the voltage of a sectional electroelastic engine, $\Delta l_{1}$ is the deformation of the first section engine and $\mathrm{c}$ is the decimal code. In the work, the mechanical and adjustment characteristics of a sectional electroelastic engine are obtained.

\section{Transient Characteristic of a Sectional Electroelastic Engine at Elastic-Inertial Load}

Let us consider the dynamic characteristics of a sectional electroelastic engine for nanomechatronics systems. The transfer function of an electroelastic engine is determined under elastic-inertial load. The ordinary linear differential equation second order of an electroelastic engine [8-10,19-27] has the form

$$
\begin{gathered}
\frac{d^{2} \Xi(x, s)}{d x^{2}}-\gamma^{2} \Xi(x, s)=0 \\
\gamma=s / c^{E}+\alpha
\end{gathered}
$$

where $\Xi(x, s), x, s, \gamma=s / c^{E}+\alpha, c^{E}$, and $\alpha$ are the Laplace transform of the displacement of an electroelastic engine, the coordinate, the Laplace operator, the wave propagation coefficient, the speed of sound at $E=$ const and the attenuation coefficient, respectively. The solution to this differential equation has the form

$$
\Xi(x, s)=C e^{-x \gamma}+B e^{x \gamma}
$$

where $C, B$ are the coefficients.

The coefficients are determined from the boundary conditions

$$
\begin{aligned}
& \Xi(0, s)=\Xi_{1}(s) \text { at } x=0 \\
& \Xi(l, s)=\Xi_{2}(s) \text { at } x=l
\end{aligned}
$$

The coefficients $C$ and $B$ have the form

$$
C=\frac{\Xi_{1}(s) e^{l \gamma}-\Xi_{2}(s)}{2 \operatorname{sh}(l \gamma)}, B=\frac{\Xi_{2}(s)-\Xi_{1}(s) e^{-l \gamma}}{2 \operatorname{sh}(l \gamma)}
$$

The solution to the ordinary differential equation for an electroelastic engine has the form

$$
\left.\Xi(x, s)=\frac{1}{\operatorname{sh}(l \gamma)}\left(\Xi_{1}(s) \operatorname{sh}((l-x) \gamma)+\Xi_{2}(s)\right) \operatorname{sh}(x \gamma)\right)
$$


With one fixed face of an electroelastic engine at $x=0, \Xi_{1}(s)=\Xi(0, s)=0$ the equation of the displacement has the form

$$
\Xi(x, s)=\frac{\left.\Xi_{2}(s)\right) \operatorname{sh}(x \gamma)}{\operatorname{sh}(l \gamma)}
$$

consequently

$$
\left.\frac{d \Xi(x, s)}{d x}\right|_{x=l}=\frac{\Xi_{2}(s) \gamma}{\operatorname{th}(l \gamma)}
$$

From the equation of the electroelasticity (1) for elastic-inertial load of an electroelastic engine the Laplace transform of the relative deformation at $x=l$ has the following form

$$
\left.\frac{d \Xi(x, s)}{d x}\right|_{x=l}=d_{m i} E_{m}(s)-\frac{s_{i j}^{E} M p^{2} \Xi_{2}(s)}{S_{0}}-\frac{s_{i j}^{E} C_{e} \Xi_{2}(s)}{S_{0}}
$$

where $M, C_{e}$ are the mass and the rigidity of the load, respectively, whence

$$
\frac{\Xi_{2}(s) \gamma}{\operatorname{th}(l \gamma)}+\frac{\Xi_{2}(s) s_{i j}^{E} M s^{2}}{S_{0}}+\frac{\Xi_{2}(s) s_{i j}^{E} C_{e}}{S_{0}}=d_{m i} E_{m}(p)
$$

Therefore, the transfer function on the electric field strength of an electroelastic engine has the form

$$
W_{E}(s)=\frac{\Xi_{2}(s)}{E_{m}(s)}=\frac{d_{m i}}{s_{i j}^{E} M s^{2} / S_{0}+\gamma \operatorname{cth}(l \gamma)+s_{i j}^{E} C_{e} / S_{0}}
$$

consequently

$$
W_{E}(s)=\frac{\Xi_{2}(s)}{E_{m}(s)}=\frac{d_{m i} l}{M s^{2} / C_{i j}^{E}+l \gamma c t h(l \gamma)+C_{e} / C_{i j}^{E}}
$$

where $C_{i j}^{E}$ is the rigidity of an electroelastic engine.

The transfer function on voltage of an electroelastic engine has the form

$$
W_{U}(s)=\frac{\Xi_{2}(s)}{U(s)}=\frac{d_{m i} l / \delta}{M p^{2} / C_{i j}^{E}+l \gamma \operatorname{cth}(\gamma)+C_{e} / C_{i j}^{E}}
$$

At elastic-inertial load for $M>>m$-where $M$ and $m$ are the masses of the load and the engine, respectively, with the approximation of the hyperbolic cotangent by two terms of the power series-the transfer function on the voltage for the lumped parameters of an electroelastic engine has the form

$$
W_{U}(s)=\frac{\Xi_{2}(s)}{U(s)}=\frac{d_{m i} l / \delta}{\left(1+C_{e} / C_{i j}^{E}\right)\left(T_{i j}^{2} s^{2}+2 T_{i j} \xi_{i j} s+1\right)}
$$

where $T_{i j}=\sqrt{M /\left(C_{e}+C_{i j}^{E}\right)}$ is the time constant and $\xi_{i j}=\alpha l^{2} C_{i j}^{E} /\left(3 c^{E} \sqrt{M\left(C_{e}+C_{i j}^{E}\right)}\right)$ is the coefficient attenuation for an electroelastic engine.

Therefore, the transfer function on the voltage for the lumped parameters of a sectional electroelastic engine at elastic-inertial load for $M>>m$ on Figure 5 for nanomechatronics systems has the form

$$
k_{U}=\frac{\left(d_{m i} l_{1} / \delta\right)}{\left(1+C_{e} / C_{i j}^{E}\right)}\left(\sum_{k=1}^{N} a_{k} 2^{k-1}\right), T_{i j}=\sqrt{M /\left(C_{e}+C_{i j}^{E}\right)}, \xi_{i j}=\alpha l^{2} C_{i j}^{E} /\left(3 c^{E} \sqrt{M\left(C_{e}+C_{i j}^{E}\right)}\right),
$$


where $M$ and $m$ are the masses of the load and the sectional electroelastic engine, respectively; $\Xi(s)$ and $U(s)$ are the Laplace transforms of the displacement and the voltage, respectively; and $k_{U}, T_{i j}$, and $\xi_{i j}$ are the transfer coefficient on the voltage, the time constant and the coefficient attenuation for a sectional electroelastic engine, respectively.

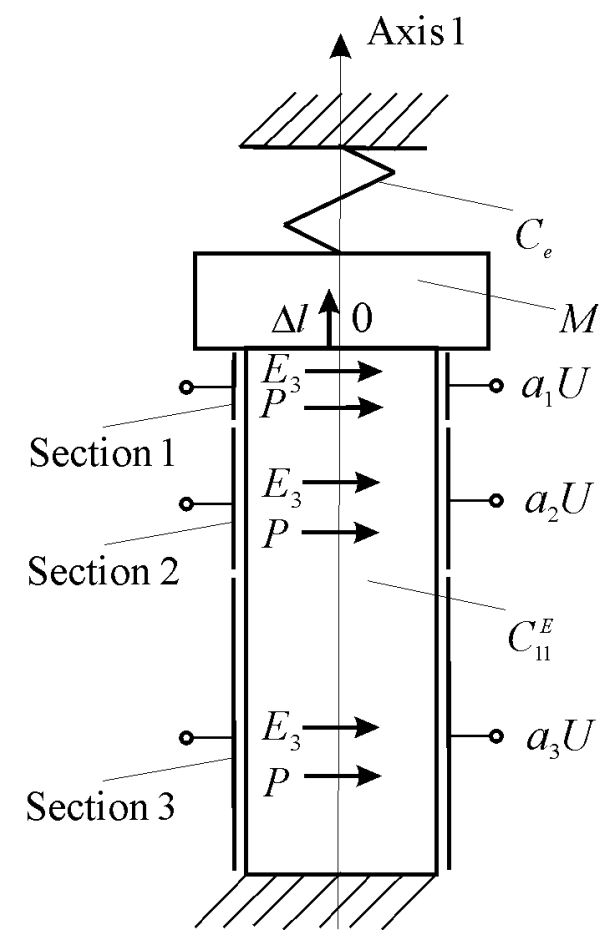

Figure 5. Sectional transverse piezoelectric engine at elastic-inertial load.

The transient characteristic for the displacement of a sectional electroelastic engine at elastic-inertial load for nanomechatronics systems from (51) is obtained in the form

$$
\begin{gathered}
\xi(t)=\xi_{0} h(t)=\xi_{0}\left(1-\frac{\sin \left(\beta_{i j} t+\varphi_{i j}\right)}{\sqrt{1-\xi_{i j}^{2}}} e^{-\frac{\xi_{i j} t}{T_{i j}}}\right) \\
\xi_{0}=k_{U} U_{0}, h(t)=1-\frac{\sin \left(\beta_{i j} t+\varphi_{i j}\right)}{\sqrt{1-\xi_{i j}^{2}}} e^{-\frac{\xi_{i j} t}{T_{i j}}}, \beta_{i j}=\frac{\sqrt{1-\xi_{i j}^{2}}}{T_{i j}}=, \varphi_{i j}=\operatorname{arctg}\left(\frac{\sqrt{1-\xi_{i j}^{2}}}{\xi_{i j}}\right)
\end{gathered}
$$

where $t, \xi_{0}, U_{0} \beta_{i j}$, and $\varphi_{i j}$ are the time, the amplitude displacement, the amplitude voltage, the natural oscillation frequency, and the initial phase of oscillation, respectively. At $k_{U}=1.1 \mathrm{~nm} / \mathrm{V}, U_{0}=100 \mathrm{~V}, M=4 \mathrm{~kg}, C_{11}^{E}=2 \times 10^{7} \mathrm{~N} / \mathrm{m}$, and $C_{e}=0.5 \times 10^{7} \mathrm{~N} / \mathrm{m}$ for a sectional transverse piezoelectric engine from ceramic PZT, $\xi_{0}=110 \mathrm{~nm}$ and $T_{t}=0.4 \times 10^{-3} \mathrm{~s}$ are obtained. The theoretical and practical parameters of a sectional piezoelectric engine are coincidences with an error of $10 \%$.

Therefore, the transfer function and the transient characteristic of a sectional electroelastic engine at elastic-inertial load are received for nanomechatronics systems.

\section{Results and Discussion}

In this work, the static and dynamic characteristics of a multilayer sectional electroelastic engine for nanomechatronics systems are obtained. The transfer function of a sectional electroelastic engine is determined under elastic-inertial load.

In space equipments including the Space Interferometer Mission are increasingly seeking to use a multilayer piezoelectric stack engines for precision positioning to accuracies of the order of fractions of a nanometer. The effective piezoelectric constant $d_{33 e f f}$ is 
determined by summing the displacement for each element of the stack and is found in the form

$$
d_{33 e f f}=n d_{33}
$$

where $n$ is the number layers of a multilayer piezoelectric stack engine and $d_{33}$ is the longitudinal piezomodule. Therefore, a low voltage of about $60 \mathrm{~V}$ is used to control a multilayer piezoelectric stack engine for driving these precision mechanisms. For control, layers can be combined into sections [6].

Multilayer structures have been introduced in the piezoelectric engine because of the following advantages: much lower engine voltage, making them safer to use, and miniaturization of engine for nanomechatronics systems. The piezolayers of multilayer structure can be combined into sections. Splitting into sections allows to use sections independently in the piezoelectric engine. The voltage of about $100 \mathrm{~V}$ is applied to a piezolayer [7]. A multilayer sectional piezoelectric engine solves tasks of precise nanopositioning adjustment for nanomechatronics systems in nanotechnology $[7,29]$.

In this study, we obtained a generalized mechanical and adjustment characteristics of a multilayer sectional electroelastic engine for nanomechatronics systems using the equations of the electroelasticity and the mechanical load. For the transverse, longitudinal, and shift piezoeffects, the mechanical and adjustment characteristics of a sectional electroelastic engine are obtained. In nanomechatronics systems, a sectional electroelastic engine is used for coded control as a digital-to-analog converter. The transfer function and the transient characteristic of a sectional electroelastic engine at elastic-inertial load are received for nanotechnology equipment.

\section{Conclusions}

The mechanical and adjustment characteristics of a sectional electroelastic engine for nanomechatronics systems are determined in this study. A sectional electroelastic engine is used as a digital-to-analog converter-engine for nanodisplacement. The dynamic characteristics of a sectional electroelastic engine at elastic-inertial load are obtained. The transfer function and the transfer coefficient of a sectional electroelastic engine at elasticinertial load for nanomechatronics systems are determined.

For the transverse, longitudinal, and shift piezoeffects, the mechanical and adjustment characteristics of a sectional electroelastic engine are received for precise nanopositioning adjustment in nanotechnology, adaptive optics, and antennas. The expression of the transient characteristic for the displacement of a sectional electroelastic engine at elasticinertial load is obtained for nanomechatronics systems.

Funding: This research received no external funding.

Institutional Review Board Statement: Not applicable.

Informed Consent Statement: Not applicable.

Data Availability Statement: Not applicable.

Conflicts of Interest: The authors declare no conflict of interest.

\section{References}

1. Schultz, J.; Ueda, J.; Asada, H. Cellular Actuators; Butterworth-Heinemann Publisher: Oxford, UK, 2017 ; p. 382.

2. Afonin, S.M. Absolute stability conditions for a system controlling the deformation of an elecromagnetoelastic transduser. Dokl. Math. 2006, 74, 943-948. [CrossRef]

3. Zhou, S.; Yao, Z. Design and optimization of a modal-independent linear ultrasonic motor. IEEE Trans. Ultrason. Ferroelectr. Freq. Control 2014, 61, 535-546. [CrossRef] [PubMed]

4. Uchino, K. Piezoelectric Actuator and Ultrasonic Motors; Kluwer Academic Publishers: Boston, MA, USA, 1997 ; p. 347.

5. Ueda, J.; Secord, T.; Asada, H.H. Large effective-strain piezoelectric actuators using nested cellular architecture with exponential strain amplification mechanisms. IEEE/ASME Trans. Mechatron. 2010, 15, 770-782. [CrossRef]

6. Sherrit, S.; Jones, C.; Aldrich, J.; Blodget, C.; Bao, X.; Badescu, M.; Bar-Cohen, Y. Multilayer piezoelectric stack actuator characterization. Proc. SPIE-Int. Soc. Opt. Eng. 2008, 6929, 692909. [CrossRef] 
7. Uchino, K. Multilayer technologies for piezoceramic materials. In Advanced Piezoelectric Materials: Science and Technology; Uchino, K., Ed.; Woodhead Publishing in Materials: Cambridge, UK, 2017; Chapter 11; pp. 423-451. [CrossRef]

8. Afonin, S.M. Block diagrams of a multilayer piezoelectric motor for nano- and microdisplacements based on the transverse piezoeffect. J. Comput. Syst. Sci. Int. 2015, 54, 424-439. [CrossRef]

9. Afonin, S.M. Structural parametric model of a piezoelectric nanodisplacement transduser. Dokl. Phys. 2008, 53, 137-143. [CrossRef]

10. Afonin, S.M. Solution of the wave equation for the control of an elecromagnetoelastic transduser. Dokl. Math. 2006, 73, 307-313. [CrossRef]

11. Cady, W.G. Piezoelectricity: An Introduction to the Theory and Applications of Electromechancial Phenomena in Crystals; McGraw-Hill Book Company: New York, NY, USA; London, UK, 1946; p. 80.

12. Mason, W.P. Methods and devices. In Physical Acoustics: Principles and Methods; Mason, W., Ed.; Academic Press: New York, NY, USA, 1964; Volume 1, Part A; p. 515.

13. Zwillinger, D. Handbook of Differential Equations; Academic Press: Boston, MA, USA, 1989; p. 673.

14. Afonin, S.M. Structural-parametric model and transfer functions of electroelastic actuator for nano- and microdisplacement. In Piezoelectrics and Nanomaterials: Fundamentals, Developments and Applications; Parinov, I.A., Ed.; Nova Science: New York, NY, USA, 2015; Chapter 9; pp. 225-242.

15. Afonin, S.M. A structural-parametric model of electroelastic actuator for nano- and microdisplacement of mechatronic system. In Advances in Nanotechnology; Bartul, Z., Trenor, J., Eds.; Nova Science: New York, NY, USA, 2017; Volume 19, Chapter 8; pp. 259-284.

16. Afonin, S.M. Nano- and micro-scale piezomotors. Russ. Eng. Res. 2012, 32, 519-522. [CrossRef]

17. Afonin, S.M. Elastic compliances and mechanical and adjusting characteristics of composite piezoelectric transducers. Mech. Solids 2007, 42, 43-49. [CrossRef]

18. Afonin, S.M. Static and dynamic characteristics of a multi-layer electroelastic solid. Mech. Solids 2009, 44, 935-950. [CrossRef]

19. Afonin, S.M. Static and dynamic characteristics of multilayered electromagnetoelastic transducer of nano- and micrometric movements. J. Comput. Syst. Sci. Int. 2010, 49, 73-85. [CrossRef]

20. Afonin, S.M. Structural-parametric model electromagnetoelastic actuator nanodisplacement for mechatronics. Int. J. Phys. 2017, 5, 9-15. [CrossRef]

21. Afonin, S.M. Structural-parametric model multilayer electromagnetoelastic actuator for nanomechatronics. Int. J. Phys. 2019, 7, 50-57. [CrossRef]

22. Afonin, S.M. Structural-parametric model of electromagnetoelastic actuator for nanomechanics. Actuators 2018, 7, 6. [CrossRef]

23. Afonin, S.M. Structural-parametric model and diagram of a multilayer electromagnetoelastic actuator for nanomechanics. Actuators 2019, 8, 52. [CrossRef]

24. Afonin, S.M. A block diagram of electromagnetoelastic actuator nanodisplacement for communications systems. Trans. Netw. Commun. 2018, 6, 1-9. [CrossRef]

25. Afonin, S.M. Decision matrix equation and block diagram of multilayer electromagnetoelastic actuator micro and nanodisplacement for communications systems. Trans. Netw. Commun. 2019, 7, 11-21. [CrossRef]

26. Afonin, S.M. Condition absolute stability control system of electromagnetoelastic actuator for communication equipment. Trans. Netw. Commun. 2020, 8, 8-15. [CrossRef]

27. Afonin, S.M. A Block diagram of electromagnetoelastic actuator for control systems in nanoscience and nanotechnology. Trans. Mach. Learn. Artif. Intell. 2020, 8, 23-33. [CrossRef]

28. Afonin, S.M. Optimal control of a multilayer electroelastic engine with a longitudinal piezoeffect for nanomechatronics systems. Appl. Syst. Innov. 2020, 3, 53. [CrossRef]

29. Bhushan, B. Springer Handbook of Nanotechnology; Springer: Berlin, Germany; New York, NY, USA, $2004 ;$ p. 1222. 